\title{
The Structure and Properties of Boron-Very-Rich Boron Carbides: B12 Icosahedra Linked through Bent CBB Chains
}

Xiaokun Yang, William A. Goddard, and Qi An

J. Phys. Chem. C, Just Accepted Manuscript • DOI: 10.1021/acs.jpcc.7b11767 • Publication Date (Web): 03 Jan 2018

Downloaded from http://pubs.acs.org on January 4, 2018

\section{Just Accepted}

"Just Accepted" manuscripts have been peer-reviewed and accepted for publication. They are posted online prior to technical editing, formatting for publication and author proofing. The American Chemical Society provides "Just Accepted" as a free service to the research community to expedite the dissemination of scientific material as soon as possible after acceptance. "Just Accepted" manuscripts appear in full in PDF format accompanied by an HTML abstract. "Just Accepted" manuscripts have been fully peer reviewed, but should not be considered the official version of record. They are accessible to all readers and citable by the Digital Object Identifier (DOI®). "Just Accepted" is an optional service offered to authors. Therefore, the "Just Accepted" Web site may not include all articles that will be published in the journal. After a manuscript is technically edited and formatted, it will be removed from the "Just Accepted" Web site and published as an ASAP article. Note that technical editing may introduce minor changes to the manuscript text and/or graphics which could affect content, and all legal disclaimers and ethical guidelines that apply to the journal pertain. ACS cannot be held responsible for errors or consequences arising from the use of information contained in these "Just Accepted" manuscripts. 


\title{
The Structure and Properties of Boron-Very-Rich Boron Carbides: $B_{12}$ Icosahedra Linked through Bent CBB Chains
}

\author{
Xiaokun Yang, ${ }^{1}$ William A. Goddard III, ${ }^{2}$ and Qi An ${ }^{1 *}$ \\ ${ }^{1}$ Department of Chemical and Materials Engineering, University of Nevada, Reno, Reno, Nevada \\ 89557, United States \\ ${ }^{2}$ Materials and Process Simulation Center, California Institute of Technology, Pasadena, \\ California 91125, United States \\ *Corresponding author E-mail: qia@unr.edu
}

\begin{abstract}
The atomic structures of boron carbide in the regime below $\sim 13.3$ at.\% $\mathrm{C}$ (known as boron-veryrich boron carbide, BvrBC) have not previously been reported due to the complexity of the structure and bonding. We report here the atomistic crystal structures for stoichiometry $\mathrm{B}_{14} \mathrm{C}$, with only 6.7 at.\% $\mathrm{C}$, predicted using quantum mechanics (QM) at the PBE level. We find that $\mathrm{B}_{14} \mathrm{C}$ consists of one $\mathrm{B}_{12}$ icosahedral cluster and one $\mathrm{C}-\mathrm{B}-\mathrm{B}$ chain per unit cell. The $\mathrm{C}-\mathrm{B}-\mathrm{B}$ chain can be linear or bent, leading to two different space groups for $\left(\mathrm{B}_{12}\right) \mathrm{CBB}$. Our bonding analyses show that both structures satisfy the electron counting rule (Wade's rule). However, the bent CBB chain which has lower crystal symmetry leads to an energy substantially more stable $(0.315$ eV per molecular unit) than the linear CBB chain structure, which has high crystal symmetry. This is because the bent CBB chain structure requires only one three-center-two-electron (3c-2e) bond while linear $\mathrm{CBB}$ chain structure requires three $3 \mathrm{c}-2 \mathrm{e}$ bonds. We predicted the mechanical properties of both structures from QM simulations. We found that shearing the linear CBB chain structure transforms first to the bent $\mathrm{CBB}$ chain structure under both pure and biaxial shear deformations. As the shear proceeds the icosahedra deconstruct due to the interaction of the CBB chains with the icosahedra. This suggests that the bent $\mathrm{CBB}$ structure is responsible for the failure processes of $\mathrm{B}_{14} \mathrm{C}$.
\end{abstract}




\section{Introduction}

Because of its superior properties of low density, ultra-high hardness, good thermal stability, high neutron absorption and low material costs, boron carbide is an attractive material for a wide range of engineering applications including body armor, abrasive powder and neutron radiation absorbent. ${ }^{1-6}$ Boron carbide is unique among engineering ceramics, consisting of 12-atom boron rich icosahedra connected via 3-atom chains into a rhombohedral unit formally referred to as $\mathrm{B}_{12} \mathrm{C}_{3}$ or more briefly as $\mathrm{B}_{4} \mathrm{C}^{6,7}$ The most stable form is written $\left(\mathrm{B}_{11} \mathrm{Cp}\right)(\mathrm{CBC})$ to indicate that there is one $\mathrm{C}$ per icosahedron (in a polar position so that it bonds directly to a $\mathrm{B}$ of another icosahedron) with one 3 atom $\mathrm{CBC}$ chain per cell connecting the icosahedra (each chain connects at 6 points to icosahedra). However, depending on the synthesis conditions, boron carbide leads to a wide composition range from 8 to 20 at.\% $\mathrm{C}^{5,8-11}$ with varying distributions of boron and carbon atoms into the icosahedra and chains of the rhombohedral crystalline lattice. This wide composition range significantly affects the physical and mechanical properties of boron carbide. ${ }^{5,8,10,12-14}$ Elemental boron also exhibits B12 icosahedra in several stable crystalline forms. The bonding within the icosahedra is considered to be strongest when there are 26 electrons available to form 13 strong delocalized multicenter intra-icosahedral bonds (Wade's rule). This pattern persists for the alloys with $\mathrm{C}, \mathrm{P}, \mathrm{N}, \mathrm{O}, \mathrm{Si}$, leading to quite complex atomistic structures.

Extensive studies ${ }^{3,5,7,15-19}$ have been aimed at determining the atomic structures of boron carbides, but the only identified structure has stoichiometry $\mathrm{B}_{4} \mathrm{C}$ leading to $\left(\mathrm{B}_{11} \mathrm{C}_{\mathrm{p}}\right) \mathrm{CBC}$ as most stable. ${ }^{20-24}$ For boron carbides richer in B, some carbon atoms must be replaced by boron atoms, leading to a range of observed stoichiometries from $\mathrm{B}_{4} \mathrm{C}$ to $\mathrm{B}_{10.2} \mathrm{C},{ }^{8}$ but the precise occupancies of the excess boron in the $\mathrm{B}_{4} \mathrm{C}$ lattices remains unknown. However, polarization and lattice distortions of the boron-rich boron carbides suggest that the substitution of carbon atoms by 
boron is not fully random. As summarized by Cheng et al. ${ }^{8}$ the carbon atoms can be replaced by boron in two ways,:

- replacement of C-B-C chains by C-B-B chains and

- replacement of $\mathrm{B}_{11} \mathrm{C}_{\mathrm{p}}$ icosahedra with $\mathrm{B}_{12}{ }^{25-27}$

Another possible structure proposed by Yakel et al. and examined by Shirai et al. is the replacement of C-B-C chains and/or C-B-B chains by a planar inter-icosahedron linking component $\left(\mathrm{B}_{4}\right)$ groups. $^{28,29}$

Several theoretical and experimental studies have suggested that the atomic bonding, electron density, mechanical properties, and lattice constants of boron carbide change significantly with boron/carbon ratios. ${ }^{16,20,30-33}$ The $\left(\mathrm{B}_{11} \mathrm{C}\right)$ icosahedron in $\mathrm{B}_{4} \mathrm{C}$ satisfies Wade's rule ${ }^{34,35}$ (26 e within icosahedron) by transferring one electron from the C-B-C chain to form a formal C-(B+)C closed shell chain. ${ }^{36}$ This seems unlikely for boron rich boron carbide. Thus, $\left(\mathrm{B}_{13} \mathrm{C}_{2}\right)$ with the structure $\left(\mathrm{B}_{12}\right)(\mathrm{CBC})$ would only have $25 \mathrm{e}$ within the icosahedron, which might reduce symmetry or cause distortion of the structure. One recent study suggests possible fractional intericosahedral bonds. ${ }^{37}$ Others found that excess boron substitution leads to expanded lattice constants, orientational asymmetry of the chain structure, and distortion of the icosahedra of rhombohedra units (compared with conventional $\mathrm{B}_{4} \mathrm{C}$ carbon-rich boron carbides). ${ }^{8,38}$ In addition, the hardness and modulus of boron-rich boron carbides measured by nanoindentation decrease with the increase of boron content, except for the $B_{10.2} C$ sample. ${ }^{8}$ To make progress in tailoring these materials for new applications, it is essential to understand the structures, chemical bonding and mechanical properties in boron carbide at boron very rich regime. 
In the present study, we apply quantum mechanics (QM) simulations to predict two configurations for stoichiometry $\mathrm{B}_{14} \mathrm{C}$ with 6.7 at.\% $\mathrm{C}$ :

- the linear chain $\left(\mathrm{B}_{12}\right) / \mathrm{CBB}$, which we find to have a positive enthalpy of formation $(+0.305$ $\mathrm{eV}$ ) with respect to $\alpha-\mathrm{B}_{12}$ and graphite

- the bent chain $\left(\mathrm{B}_{12}\right) \mathrm{bCBB}$, which we find to have a negative enthalpy of formation $(-0.010$ $\mathrm{eV}$ ) with respect to $\alpha-\mathrm{B}_{12}$ and graphite, suggesting it can be synthesized experimentally.

Then we analyzed the bonding for both configurations, finding that both configurations satisfy Wade's electron counting rule. Finally, we examined their mechanical properties by deriving the stress-strain relationships, the ideal shear strength, and the failure mechanisms under pure shear deformation and biaxial shear deformation.

\section{Computational Methods}

All QM calculations were performed with the VASP package, ${ }^{39-41}$ which applies periodic boundary conditions and uses plane wave basis functions. We used the Perdew-Burke-Ernzerhof (PBE) functional to account for the electron exchange-correlation interactions and the projector augmented wave method to account for core-valence interactions. ${ }^{42}$ The energy cutoff for the plane wave expansion was $600 \mathrm{eV}$. The energy error for terminating electronic self-consistent field (SCF) and the force criterion for the geometry optimization were set equal to $10^{-6} \mathrm{eV}$ and $10^{-3} \mathrm{eV} / \AA$, respectively. The Brillouin zone integration was performed on $\Gamma$-centered symmetryreduced Monkhorst-Pack meshes with a fine resolution of $2 \pi \times 1 / 40 \AA^{-1}$ for all calculations except for the shear deformation. A more approximate $2 \times 2 \times 2$ k-point grid mesh in the Brillouin zone was applied for both pure shear and biaxial shear deformation. 
We performed ab initio molecular dynamics (AIMD) simulations for $2 \times 2 \times 2$ supercells to examine the stability of predicted structures. These systems were equilibrated at finite temperatures for $10 \mathrm{ps}$ using the NVT (constant volume, constant temperature and constant number of atoms) ensemble. We used a time step of 1.0 femtosecond for integrating the equations of motion.

To understand the chemical bonding in the two structures, we performed electron localization function $(E L F)^{43,44}$ analyses to identify the two-center-two-electron (2c-2e) and threecenter-two-electron (3c-2e) bonds. To provide spectroscopic signatures to distinguish between these two structures, we calculated the off-resonance Raman intensity by computing the derivative of the polarizability (or macroscopic dielectric tensor) with respect to that normal mode coordinate. The phonons at $\Gamma$-point and the macroscopic dielectric tensor were both calculated using density functional perturbation theory (DFPT) as implemented in VASP.

To predict the mechanical properties of the two configurations, we computed their elastic constants, bulk modulus, shear modulus, and Pugh's ductility index $(B / G)$. The elastic constant $\mathrm{C}_{\mathrm{ij}}$ were derived from the stress-strain relation as a function of various cell distortions from the equilibrium lattice configuration. ${ }^{45}$ The isotropic polycrystalline bulk and shear modulus were computed using the Voigt-Reuss-Hill (VRH) approximation. ${ }^{46}$

To determine the failure mechanisms for the $\left(\mathrm{B}_{12}\right) \mathrm{CBB}$ structures, we imposed the strain for a particular shear plane, while allowing full structure relaxation of the other five strain components. $^{47}$

To simulate the mechanical response under indentation experiments, we applied biaxial shear deformation. ${ }^{48}$ This simulation aimed at mimicking deformation under the indenter by imposing 
the relations $\sigma_{\mathrm{zz}}=\sigma_{\mathrm{zx}} \times \tan \Phi$ where $\sigma_{\mathrm{zz}}$ is the normal stress, $\sigma_{\mathrm{zx}}$ is the shear stress and $\Phi$ is the centerline-to face angle of the indenter $\left(\Phi=68^{\circ}\right.$ for the Vickers indenter $) .{ }^{48}$ The other four strain components are relaxed for the biaxial shear deformation.

The residual stresses after relaxing were $<0.5 \mathrm{GPa}$ for both pure shear and biaxial shear deformation. For these shear simulations, we used $2 \times 2 \times 2$ supercells with 120 atoms. Since the shear strain is constrained in the deformation, the stress of the system may become negative after the structure changes or fails.

\section{Results and Discussion}

Figure $1(\mathrm{a})$ and $(\mathrm{b})$ display the linear chain $\left(\mathrm{B}_{12}\right) / \mathrm{CBB}$ and bent chain $\left(\mathrm{B}_{12}\right) \mathrm{bCBB}$ crystal structures, respectively. We find that $\left(B_{12}\right) / C B B$ has the $R \overline{3} \mathrm{~m}$ space group where the $B_{12}$ icosahedral cluster is located on the corner and the C-B-B chain is along the $[111]_{\mathrm{r}}$ rhombohedral directions. Here, the subscript "r" represents the rhombohedral cell. PBE gives the optimized lattice parameters of $\mathrm{a}=5.133 \AA$ and $\alpha=66.8^{\circ}$ with a density of $2.47 \mathrm{~g} / \mathrm{cm}^{3}$ for linear chain $\left(\mathrm{B}_{12}\right) / \mathrm{CBB}$ structure. Here the $\mathrm{C} 1-\mathrm{B} 12-\mathrm{B} 14$ chain angle is $180.0^{\circ}$ and the $\mathrm{B} 12-\mathrm{C} 1$ and $\mathrm{B} 12-\mathrm{B} 14$ bond distances are 1.598 and $1.556 \AA$, respectively, suggesting that both are 2c-2e bonds.

For the bent chain $\left(\mathrm{B}_{12}\right)$ bCBB structure, crystal symmetry is lowered to P1 space group, with optimized lattice parameters of $a=5.084 \AA, b=5.140 \AA, c=5.204 \AA, \alpha=67.0^{\circ}, \beta=65.8^{\circ}$, and $\gamma=68.2^{\circ}$. This leads to a density of $2.45 \mathrm{~g} / \mathrm{cm}^{3}, 1 \%$ lower density than the linear chain structure. Here the $\mathrm{C} 1-\mathrm{B} 12-\mathrm{B} 14$ chain angle is $96.4^{\circ}$ and the B12-C1 bond distance is $1.609 \AA$ while the B12-B14 bond distance is $1.720 \AA$, suggesting quite different bonding conditions.

To determine the stability of these two structures, we computed the enthalpies of formation with respect to the stable forms of boron $\left(\alpha-B_{12}\right)$ and carbon (graphite). The computed enthalpies 
of formation for bent structure and linear structure are -0.010 and $0.305 \mathrm{eV}$ per unit cell. The enthalpy of formation for linear structure is positive which is consistent with previous QM simulations, ${ }^{13}$ suggesting that it would be difficult to synthesize as a single crystal phase. However, the more stable bent chain structure has negative heat of formation, suggesting the possibility of synthesizing a single crystal. We note here that the bent chain structure is more stable even though it has lower symmetry than the linear chain structure.

To understand why the low symmetry bent chain structure is more stable than linear chain structure, we performed the bonding analyses on both structures with the ELF analysis shown in Figure $1(\mathrm{c})$ and $(\mathrm{d})$. In linear chain $\left(\mathrm{B}_{12}\right) / \mathrm{CBB}$ structure, the chain carbon atom is bonded to the three nearby icosahedra through three $2 \mathrm{c}-2 \mathrm{e}$ bonds and to the center B12 atom through one $2 \mathrm{c}-2 \mathrm{e}$ sigma bond. Meanwhile, both B12 and B14 atoms (in the chain) are bonded to three nearby icosahedra through three 3c-2e bonds: B12-B14-B1, B12-B14-B2 and B12-B14-B3 bonds, as shown in Figure 1(c). For the triangular B3 unit formed by B12-B14-B2 the bond distances are $1.68 \AA, 2.00 \AA$ and $1.56 \AA$ for B14-B2, B12-B2 and B14-B12, respectively. This suggests that atom $\mathrm{B} 14$ provides more electrons to $3 \mathrm{c}-2 \mathrm{e}$ bonds than $\mathrm{B} 12$ and $\mathrm{B} 2$ atoms. Therefore, we can assume $\mathrm{B} 14$ atom provides $(2 / 3+\delta)(0<\delta<1 / 3)$ electrons to $3 \mathrm{c}-2 \mathrm{e}$ bonds and $\mathrm{B} 2$ and $\mathrm{B} 12$ atoms both provide $(2 / 3-\delta / 2)$ electrons to $3 c-2$ e bonds. Thus, each icosahedron contributes $(9+3 *(2 / 3-$ $\delta / 2))=(11-3 \delta / 2)$ electrons to the exo-polyhedral bond, leaving $(25+3 \delta / 2)$ electrons within the icosahedron. Meanwhile the B14 and B12 atoms contribute $(2+3 \delta)$ and $(2-3 \delta / 2)$ electrons to

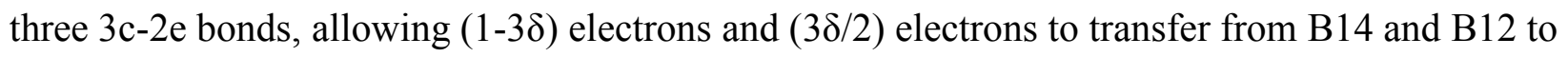
the nearby icosahedron, respectively. This makes this two chain B atoms both Lewis acids. Thus, the icosahedron has $(25+3 \delta / 2)+(1-3 \delta)+(3 \delta / 2)=26$ electrons, satisfying the Wade's rule. This leads to a representation as $\left(\mathrm{B}_{12}\right)^{1-} \mathrm{C}-\mathrm{B}^{38 / 2+}-\mathrm{B}^{(1-38)^{+}}$for the linear chain structure. The Bader 
charge analysis indicates that the charges are $+1.40,+0.08$, and -0.25 on B12, B14 and B2 atoms, respectively. This is consistent with our bonding analysis that electrons will transfer from chain B12 and B14 atoms to nearby icosahedron.

The bonding in bent chain structure can be described as follows. In the CBB bend chain, each carbon is bonded to three nearby icosahedra and the central B12 atom through 2c-2e sigma bonds. The side B14 is bonded to two nearby icosahedra through $2 \mathrm{c}-2 \mathrm{e}$ bonds and is bonded to the other icosahedron through the B12-B14-B1 3c-2e bond, as shown in Figure 1(d). The bond distances around the B12-B14-B1 ring are $1.72 \AA, 1.73 \AA$, and $1.78 \AA$ for B12-B14, B14-B1 and B12-B1, respectively. Thus, the bond distances are slightly different around 3c-2e bond and each $\mathrm{B}$ atom is approximately considered to provide $1 / 3$ electrons to the $3 \mathrm{c}-2 \mathrm{e}$ bond. This leaves $1 / 3$ electrons left on the B14 atom. The central B12 atom contributes one electron to the B12-C sigma bond and 2/3 electrons to B12-B14-B1 bonds (Figure 1(d)), leaving 4/3 electrons. For the icosahedron, it forms $112 \mathrm{c}-2 \mathrm{e}$ and one 3c-2e exo-polyhedral bonds, leaving 24+1/3 electrons within the icosahedron. Therefore, the chain electrons can be transferred to nearby icosahedron, forming $1 / 3+4 / 3+24+1 / 3=26$ electrons, satisfying the Wade's rule. In addition, the Bader charge analysis shows that the charges in B12, B14 and B1 atoms are $+1.45,-0.05$ and -0.19 , respectively. The charge analysis supports our bonding analysis that electrons will transfer from $\mathrm{B} 12$ and B14 atoms to the nearby icosahedron, respectively. This leads to a representation as $\left(B_{12}\right)^{5 / 3-} C-B^{4 / 3+}-B^{1 / 3+}$ for the bent chain structure. There is only one $3 c-2 e$ bond in the bent chain structure, while there are three in the linear chain structure. This makes the bent chain structure much more stable despite its lower symmetry. To validate that both bent and linear chain structure is stable at finite temperature, we preformed ab initio molecular dynamics (AIMD) 
simulations on both structures at room temperature. Both structures are intact after 4 picoseconds of AIMD, suggesting they neither are intrinsically unstable at finite temperature.

Because the bent chain structure is $0.315 \mathrm{eV}$ per molecular unit more stable than the linear CBB structure, it is interesting to examine the relative stability of both structures at high temperature. Here we performed the AIMD simulations and found that the linear chain $\left(\mathrm{B}_{12}\right) \mathrm{CBB}$ structure can easily transform to the bent structure when it is heated from $300 \mathrm{~K}$ to $1200 \mathrm{~K}$ within $10 \mathrm{ps}$. While starting with the bent chain structure, it remains stable at high temperature of $1200 \mathrm{~K}$ after another 10 ps simulation. Considering the CBB chain can bend in several possible planes to its nearby icosahedra, we quenched the bent chain structure from $1200 \mathrm{~K}$ to $10 \mathrm{~K}$ within 10 ps. Then we optimized the structure to find a larger unit cell with eight $\left(\mathrm{B}_{12}\right) \mathrm{CBB}$ molecules, as shown in Figure S1 of Supporting Information (SI). The CBB chains bend in different directions (Fig. S1 of SI), leading to lower energy by $0.221 \mathrm{eV}$ per molecule compared to the rhombohedral unit cell. This indicates that crystals of the bent chain structure will exhibit several bending planes so that it may remain disordered. 


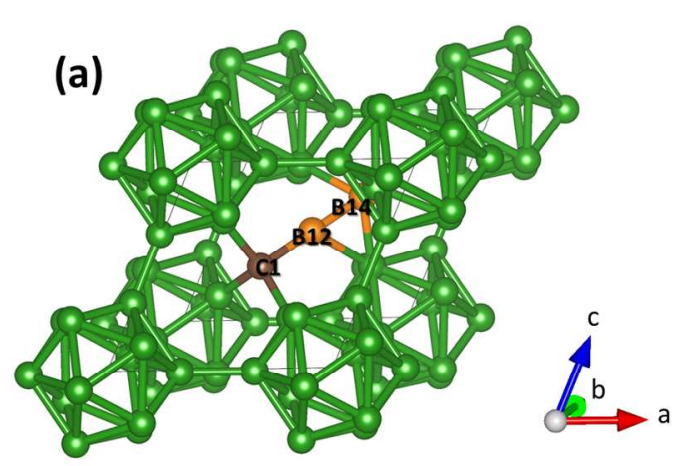

Linear chain $\left(B_{12}\right) C B B$

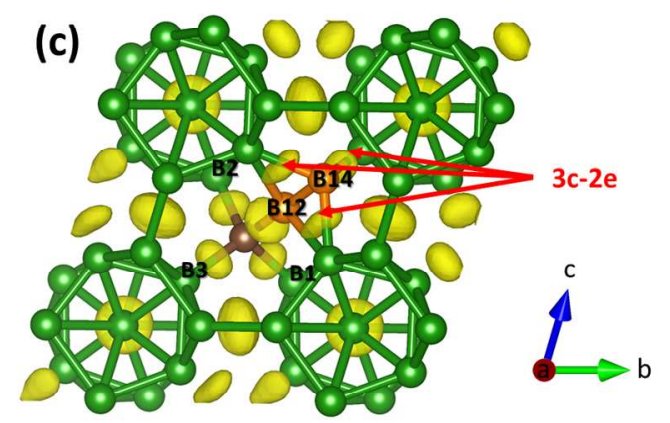

Linear chain $\left(\mathrm{B}_{12}\right) \mathrm{CBB}$
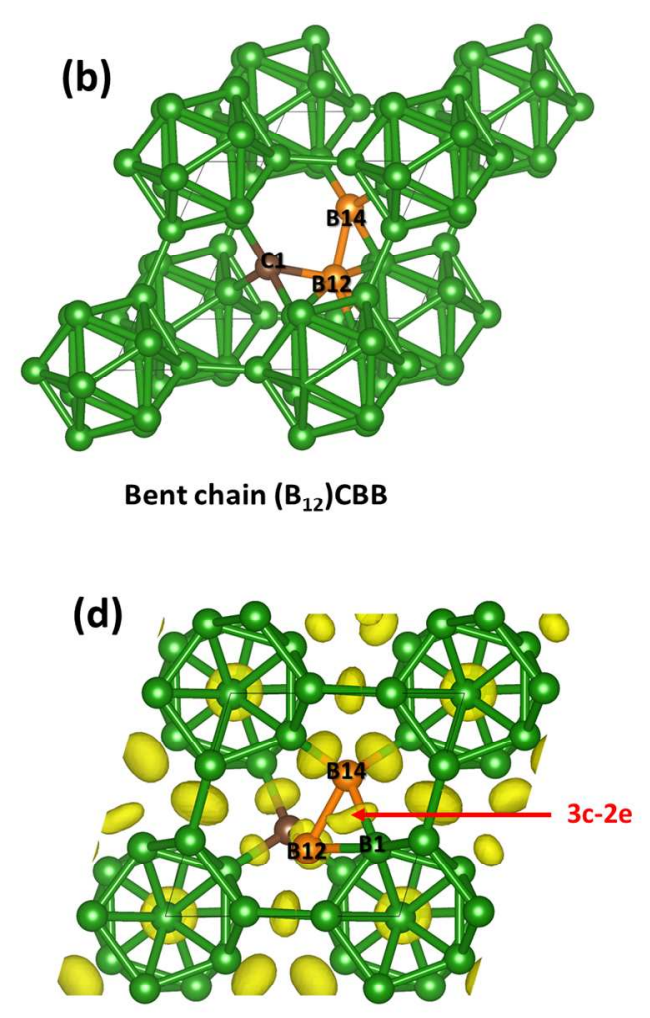

Bent chain $\left(B_{12}\right) C B B$

Figure 1. The optimized structures from DFT: (a) crystalline linear chain $\left(\mathrm{B}_{12}\right)(\mathrm{CBB})$, (b) crystalline bent chain $\left(\mathrm{B}_{12}\right)(\mathrm{bCBB})$; with the calculated ELF (isosurfaces at 0.85 ) for the structures (c) linear chain $\left(\mathrm{B}_{12}\right)(\mathrm{CBB})$ and $(\mathrm{d})$ bent chain $\left(\mathrm{B}_{12}\right)(\mathrm{bCBB})$. The orange color indicates chain $\mathrm{B}$ atoms.

To provide signatures to identify experimentally which structures are prepared, we predicted the Raman spectra (Figure 2) using the QM optimized structures. In the simulated Raman spectra, the bent chain structure shows vibration modes in the lower frequency range $\left(200 \mathrm{~cm}^{-1} \sim 400 \mathrm{~cm}^{-}\right.$ ${ }^{1}$ ) while the low frequency modes for the linear structure are in the range $300 \mathrm{~cm}^{-1} \sim 400 \mathrm{~cm}^{-1}$. These low frequency modes involve the C-B-B chain movements, as shown in the movies in the SI. The linear C-B-B chain shows both stretching modes along the chain direction (frequency of $359 \mathrm{~cm}^{-1}$ ) and bending modes (frequencies of $338.6 \mathrm{~cm}^{-1}$ and $394.8 \mathrm{~cm}^{-1}$ ), while the bent chain shows only bending modes (frequencies of $184.4 \mathrm{~cm}^{-1}$ and $312.3 \mathrm{~cm}^{-1}$ ). The difference in the Raman active peaks should allow the chain geometries to be assigned experimentally. 
The $\mathrm{B} / \mathrm{C}$ ratio has a significant effect on the elastic modulus of boron carbide samples. ${ }^{8}$ It has been suggested that the expanded lattices of boron rich boron carbide caused by boron replacement is responsible for the reduced strength. ${ }^{33}$ The calculated bulk modulus for linear and bent chain $\left(\mathrm{B}_{12}\right) \mathrm{CBB}$ are 197.5 and $212.3 \mathrm{GPa}$, respectively. The predicted shear moduli are 167.1 and 188.7 GPa for linear and bent chain structures, respectively. Therefore, the bent chain structure has $7.5 \%$ and $12.9 \%$ higher bulk and shear modulus than linear chain structure. However, the bulk modulus and shear modulus for bent chain structure are still $10.8 \%$ and $5.2 \%$ lower than those of $\mathrm{B}_{4} \mathrm{C}$ ( $238 \mathrm{GPa}$ for $\mathrm{B}$ and $199 \mathrm{GPa}$ for $\left.\mathrm{G}\right){ }^{36}$ This is consistent with the experimental data for boron rich boron carbide ${ }^{20}$ indicating that the higher boron content leads to lower elastic moduli. The Pugh's ductility indexes B/G for linear and bent chain structures are 1.18 and 1.12 , suggesting that both structures are brittle.

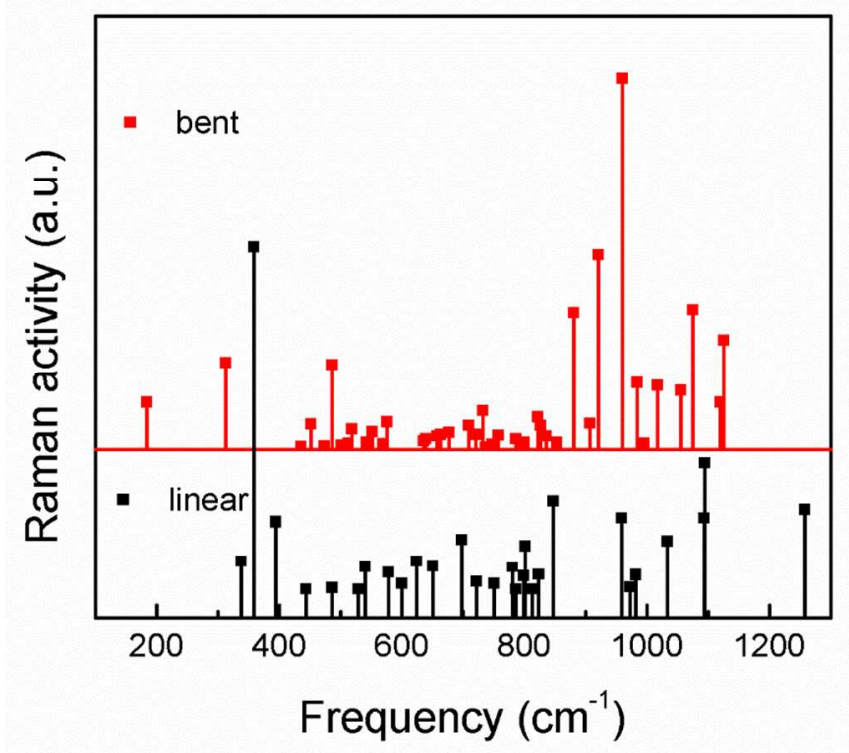

Figure 2. The simulated Raman spectra based on the structures from QM simulations of linear chain $\left(\mathrm{B}_{12}\right) \mathrm{CBB}$ and bent chain $\left(\mathrm{B}_{12}\right) \mathrm{CBB}$.

To investigate the brittle failure processes of $\left(\mathrm{B}_{12}\right) \mathrm{CBB}$ structures, we applied pure shear deformation on the linear chain structure along the most plausible slip system $(001) /<100>$ in $\mathrm{B}_{4} \mathrm{C} .{ }^{36} \mathrm{We}$ find that the high energy linear chain structure transforms easily to the bent chain 
structure (discussed below), again indicating an unstable structure. In addition, in real materials the bent $\mathrm{CBB}$ chain can randomly bend in various planes with respect to nearby icosahedra, making the structures more complex.

This shear-stress-shear-strain relationship is displayed in Figure 3(a). The ideal shear strength for the linear chain $\left(\mathrm{B}_{12}\right) \mathrm{CBB}$ structure is $30.0 \mathrm{GPa}$, which is much lower than $\mathrm{B}_{4} \mathrm{C},{ }^{36}$ suggesting that it is weaker than $\mathrm{B}_{4} \mathrm{C}$. The deformation processes are displayed in Figure 3(b)-(e). As the shear strain increases to 0.061 , the shear stress is released by $\sim 1.5 \mathrm{GPa}$, suggesting a structural change. From the structure at 0.061 strain, we observe that the linear chain structure (Figure 3(b)) deforms to bent C-B-B chains, as shown in Figure 3(c). As the shear strain increases from 0.061 to 0.369 , the C-B-B angle increases from $100.1^{\circ}$ to $125.1^{\circ}$, as shown in Figure $3(\mathrm{~d})$. At this point the middle $\mathrm{B}$ in the $\mathrm{C}-\mathrm{B}-\mathrm{B}$ chain that was originally $1.945 \AA$ now is $1.784 \AA$ at 0.06 strain. Meanwhile, the boron atoms (B1, B13) within the icosahedra are stretched apart. The B1-B13 bond within the icosahedra increases from the original 1.765 to $2.017 \AA$ at 0.369 strain. However, the icosahedral clusters do not deconstruct yet at 0.369 strain. With further shear to 0.416 , the angle of the C-B-B chain between the icosahedra decreases slightly to $121.3^{\circ}$, and the distance between B1 and B13 atoms within icosahedra increases dramatically to $2.526 \AA$, causing the icosahedron to fracture (Figure 3(e)) while relieving the shear stress to $13.4 \mathrm{GPa}$. This failure processes suggests that brittle failure arises from the interaction between the $\left(\mathrm{B}_{12}\right)$ icosahedra and the bent C-B-B chains. 
(a)

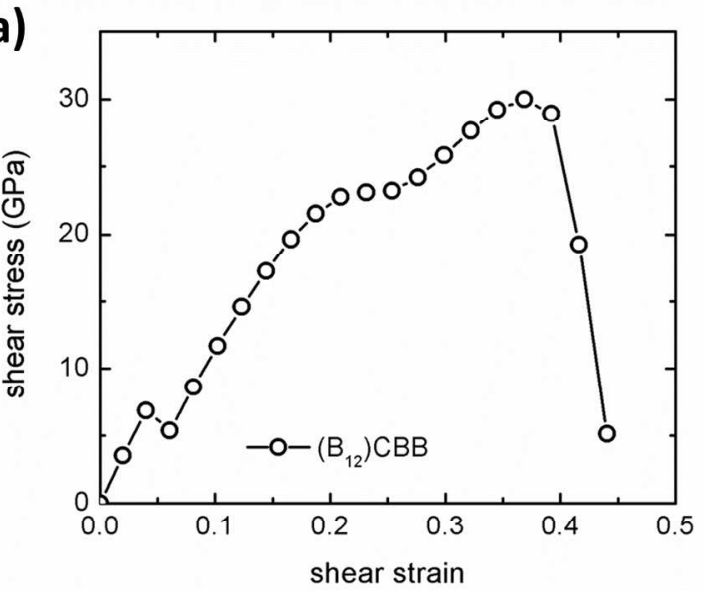

(b)

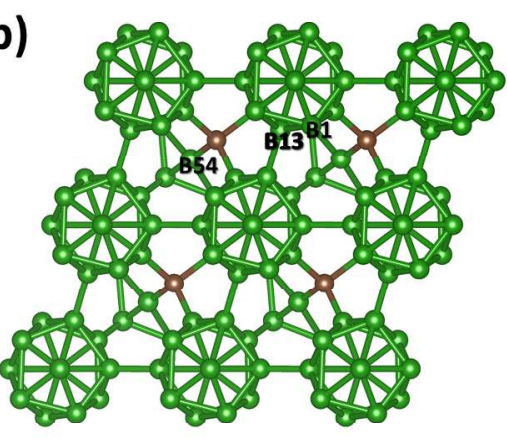

Strain $=0$

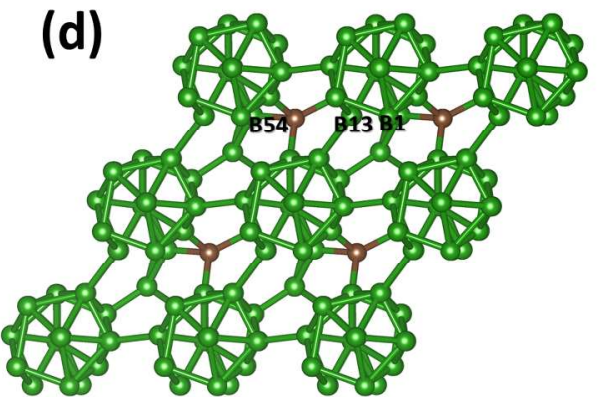

Strain $=\mathbf{0 . 3 6 9}$

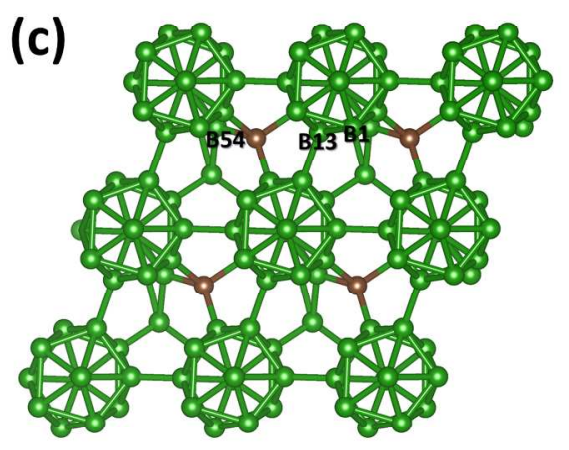

Strain $\mathbf{= 0 . 0 6 1}$

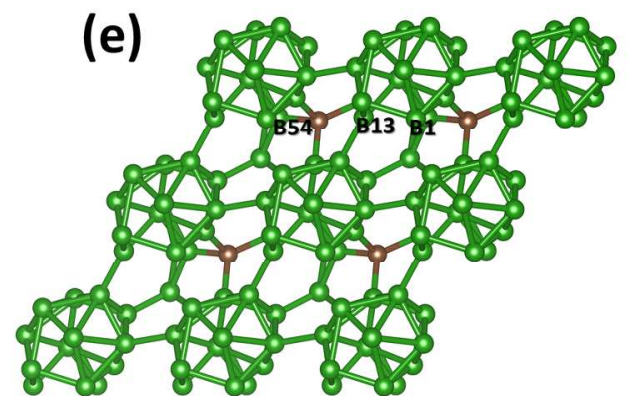

Strain $=\mathbf{0 . 4 1 6}$

Figure 3. (a) Shear-stress-shear-strain relationship of crystalline the linear chain $\left(B_{12}\right) / C B B$ structure. Structure evolution of crystalline $\left(\mathrm{B}_{12}\right) \mathrm{CBB}$ under pure shear deformation: (b) The structure at 0.0 strain; (c) The structure at 0.061 strain; (d) The structures at 0.369 strain corresponding to the maximum shear stress; (e) The failed structures at 0.416 strain.

The stress conditions under indentation experiments are very complex compared to our simulated pure shear deformation. To predict materials behavior under indentation experiments, we performed biaxial shear deformation to mimic the stress conditions under indentation. ${ }^{48}$ The 
shear-stress-shear-strain relationships of the crystalline $\left(\mathrm{B}_{12}\right)$ lCBB are shown in Figure $4(\mathrm{a})$. The maximum shear stress for $\left(\mathrm{B}_{12}\right) \mathrm{CBB}$ is $20.8 \mathrm{GPa}$, lower than crystalline $\mathrm{B}_{13} \mathrm{C}_{2}(28.6 \mathrm{GPa})^{49}$ and $\mathrm{B}_{4} \mathrm{C}(28.5 \mathrm{GPa}){ }^{49}$ This result suggests that the hardness of $\left(\mathrm{B}_{12}\right)$ CBB should be lower than $\mathrm{B}_{4} \mathrm{C}$, which is consistent with the experimental observatin. ${ }^{8}$

The detailed deformation processes of $\left(\mathrm{B}_{12}\right) \mathrm{CBB}$ under biaxial shear deformation are displayed in Figure 4(b-d). Similar to the situation for pure shear, the linear C-B-B chains deform into more stable bent chains as the strain increases from 0.02 to 0.04 , as shown in Figure 4(b). As strain increases from 0.04 to 0.254 , the middle boron atom B54 inserts into to the nearby icosahedron (Figure 4(c)). The B54-B70 bond distance increases from 1.556 to $1.645 \AA$. As the shear strain increases further to 0.276 , all of the icosahedra are deconstructed (Figure 4(d)). At 0.276 strain, the chain bond (B70-B54) is significantly stretched from to $2.792 \AA$, and one boron atom (B77) within the icosahedron is pulled out from the cage, leading to full deconstruction of the icosahedra in the $\left(\mathrm{B}_{12}\right) \mathrm{CBB}$ structure.
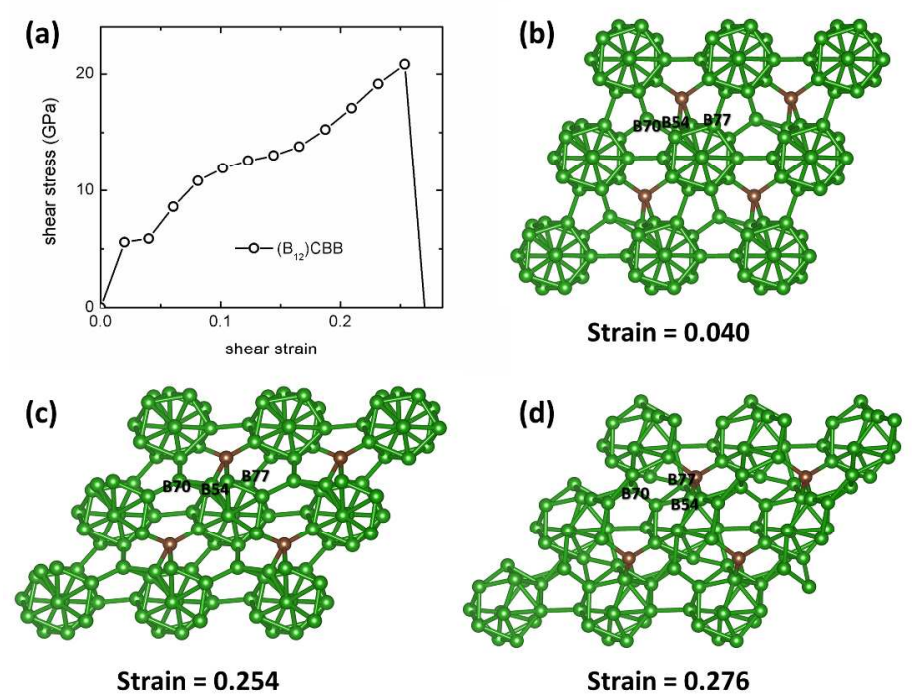

Figure 4. (a) Shear-stress-shear-strain relationship for linear chain crystalline $\left(\mathrm{B}_{12}\right) / \mathrm{CBB}$ under indentation stress condition and structural changes: (b) structure at 0.040 strain before failure; (c) structure at 0.155 strain before failure; (d) failed structure at 0.173 strain. 


\section{Summary}

In summary, we used QM simulations to predict the atomistic structures for two forms of $\mathrm{B}_{14}$ Cwith just 6.7 at. $\% \mathrm{C}$.

- We predicted the structures and properties for both linear chain $\left(\mathrm{B}_{12}\right) / \mathrm{CBB}$ and bent chain $\left(\mathrm{B}_{12}\right) \mathrm{bCBB}$ structures. The bonding analyses show that there is one $3 \mathrm{c}-2 \mathrm{e}$ bonds in the unit cell of the bent chain structure while there are three in linear chain structure. This makes bent chain structure much more stable.

- Comparing the mechanical properties of these two configurations, we find that the bent chain $\left(\mathrm{B}_{12}\right) \mathrm{bCBB}$ has a $7.5 \%$ higher bulk modulus and slightly $(1 \%)$ lower density than the linear chain structure. The linear chain structure transforms easily to bent structure as the shear is applied, suggesting that it is far less stable.

- Under pure shear deformation the boron very rich $\left(\mathrm{B}_{12}\right) \mathrm{CBB}$ structure is predicted to have an ideal shear strength comparable to that of boron rich boron carbide $\left(\mathrm{B}_{13} \mathrm{C}_{2}\right)$. We find that the failure mechanism for $\left(\mathrm{B}_{12}\right) \mathrm{CBB}$ structure involves the interaction of the $\mathrm{C}-\mathrm{B}-\mathrm{B}$ chains with the icosahedral clusters, just as in $\mathrm{B}_{4} \mathrm{C}$

- Under biaxial shear conditions, the ideal shear strength of the boron very rich $\left(\mathrm{B}_{12}\right) \mathrm{CBB}$ structure is lower than that of boron rich boron carbide $\left(\mathrm{B}_{13} \mathrm{C}_{2}\right)$. This arises from the difference in the failure mechanism under indentation loading conditions where the icosahedra in the $\left(\mathrm{B}_{12}\right) \mathrm{CBB}$ are fully deconstructed after one chain $\mathrm{B}$ atom inserts into the icosahedra and one B atom is pulled out.

\section{Supporting Information}


Figure for optimized $\left(\mathrm{B}_{12}\right) \mathrm{CBB}$ structure after AIMD simulations, and movies of vibration modes at different Raman frequencies for bent and linear chain $\left(\mathrm{B}_{12}\right) \mathrm{CBB}$ structure. The movies were named by liner or bent to indicate the linear chain $\left(\mathrm{B}_{12}\right)(/ \mathrm{CBB})$ or bent chain $\left(\mathrm{B}_{12}\right)(\mathrm{bCBB})$ structures, and followed with number of frequency $\left(\mathrm{cm}^{-1}\right)$ to indicate different vibrations. This material is available free of charge via the Internet at http://pubs.acs.org.

\section{Acknowledgments}

This work was supported by the National Science Foundation (CMMI-1727428). In addition, WAG received the support from the Defense Advanced Research Projects Agency (W31P4Q-131-0010 and W31P4Q-12-1-0008).

\section{Reference}

(1) Chen, M. W.; McCauley, J. W.; Hemker, K. J. Shock-Induced Localized Amorphization in Boron Carbide. Science 2003, 299, 1563-1566.

(2) Reddy, K. M.; Liu, P.; Hirata, A.; Fujita, T.; Chen, M. W. Atomic Structure of Amorphous Shear Bands in Boron Carbide. Nat. Commun. 2013, 4, 2483.

(3) Vast, N.; Sjakste, J.; Betranhandy, E. Boron Carbides from First Principles. J. Phys. Conf. Ser. 2009, 176, 12002.

(4) Domnich, V.; Reynaud, S.; Haber, R. A.; Chhowalla, M. Boron Carbide: Structure, Properties, and Stability under Stress. J. Am. Ceram. Soc. 2011, 94, 3605-3628.

(5) Thevenot, F. Boron Carbide-A Comprehensive Review. J. Eur. Ceram. Soc. 1990, 6, 205225.

(6) Clark, H. K.; Hoard, J. L. The Crystal Structure of Boron Carbide. J. Am. Chem. Soc. 
1943, 65, 2115-2119.

(7) Hoard, J. L.; Hughes, R. E. Elemental Boron and Compounds of High Boron Content: Structure, Properties, and Polymorphism. The Chemistry of Boron and Its Compounds; E. L. Muetterties Eds.; Wiley, New York, 1967.

(8) Cheng, C.; Reddy, K. M.; Hirata, A.; Fujita, T.; Chen, M. W. Structure and Mechanical Properties of Boron-Rich Boron Carbides. J. Eur. Ceram. Soc. 2017, 37, 4514-4523.

(9) Gosset, D.; Colin, M. Boron Carbides of Various Compositions: An Improved Method for X-Rays Characterisation. J. Nucl. Mater. 1991, 183, 161-173.

(10) Bouchacourt, M.; Thevenot, F. The Properties and Structure of the Boron Carbide Phase. $J$. less common Met. 1981, 82, 227-235.

(11) Schwetz, K. A.; Karduck, P. Investigations in the Boron-Carbon System with the Aid of Electron Probe Microanalysis. J. Less Common Met. 1991, 175, 1-11.

(12) Xie, K. Y.; Livi, K.; McCauley, J. W.; Hemker, K. J. Precipitation of AlN in a Commercial Hot-Pressed Boron Carbide. Scr. Mater. 2015, 101, 95-98.

(13) Saal, J. E.; Shang, S.; Liu, Z. kui.; Saal, J. E.; Shang, S.; Liu, Z. The Structural Evolution of Boron Carbide via Ab Initio Calculations The Structural Evolution of Boron Carbide via Ab Initio Calculations. Appl. Phys. Lett. 2007, 91, 231915.

(14) Chen, M. W.; McCauley, J. W.; LaSalvia, J. C.; Hemker, K. J. Microstructural Characterization of Commercial Hot-Pressed Boron Carbide Ceramics. J. Am. Ceram. Soc. 2005, $88,1935-1942$.

(15) An, Q.; Goddard III, W. A.; Xie, K. Y.; Sim, G. D.; Hemker, K. J.; Munhollon, T.; Fatih 
Toksoy, M.; Haber, R. A. Superstrength through Nanotwinning. Nano Lett. 2016, 16, 7573-7579.

(16) Shirai, K. Electronic Structures and Mechanical Properties of Boron and Boron-Rich Crystals (Part 1). J. Superhard Mater. 2010, 32, 205-225.

(17) Shirai, K. Electronic Structures and Mechanical Properties of Boron and Boron-Rich Crystals (Part 2). J. Superhard Mater. 2010, 32, 336-345.

(18) Suri, A. K.; Subramanian, C.; Sonber, J. K.; Murthy, T. S. R. C. Synthesis and Consolidation of Boron Carbide: A Review. Int. Mater. Rev. 2010, 55, 4-40.

(19) Werheit, H.; Filipov, V.; Kuhlmann, U.; Schwarz, U.; Armbrüster, M.; Leithe Jasper, A.; Tanaka, T.; Higashi, I.; Lundström, T.; Gurin, V. N.; et al. Raman Effect in Icosahedral Boron-Rich Solids. Sci. Technol. Adv. Mater. 2010, 11, 23001.

(20) Taylor, D. E.; McCauley, J. W.; Wright, T. W. The Effects of Stoichiometry on the Mechanical Properties of Icosahedral Boron Carbide under Loading. J. Phys. Condens. Matter 2012, 24, 505402.

(21) Fanchini, G.; Mccauley, J. W.; Chhowalla, M. Behavior of Disordered Boron Carbide under Stress. Phys. Rev. Lett. 2006, 97, 35502.

(22) Feng, Y.; Seidler, G. T.; Cross, J. O.; Macrander, A. T.; Rehr, J. J. Role of Inversion Symmetry and Multipole Effects in Nonresonant X-Ray Raman Scattering from Icosahedral B4C. Phys. Rev. B 2004, 69, 125402.

(23) Vast, N.; Besson, J. M.; Baroni, S.; Corso, A. D. Atomic Structure and Vibrational Properties of Icosahedral Alpha-Boron and B4C Boron Carbide. Comput. Mater. Sci. 2000, 
$17,127-132$.

(24) Kwei, G. H.; Morosin, B. Structures of the Boron-Rich Boron Carbides from Neutron Powder Diffraction: Implications for the Nature of the Inter-Icosahedral Chains. J. Phys. Chem. 1996, 100, 8031-8039.

(25) Morosin, B.; Kwei, G. H.; Lawson, A. C.; Aselage, T. L.; Emin, D. Neutron Powder Diffraction Refinement of Boron Carbides Nature of Intericosahedral Chains. J. Alloys Compd. 1995, 226, 121-125.

(26) Tallant, D. R.; Aselage, T. L.; Campbell, A. N.; Emin, D. Boron Carbide Structure by Raman Spectroscopy. Phys. Rev. B 1989, 40, 5649-5656.

(27) Emin, D. Structure and Single-Phase Regime of Boron Carbides. Phys. Rev. B 1988, 38, $6041-6055$.

(28) Shirai, K.; Sakuma, K.; Uemura, N. Theoretical Study of the Structure of Boron Carbide B13C2. Phys. Rev. B 2014, 90, 64109.

(29) Yakel, H. L. The Crystal Structure of a Boron-Rich Boron Carbide. Acta Crystallogr. B 1975, 31, 1797-1806.

(30) Golubeva, N. A.; Plyasunkova, L. A.; Kelina, I. Y.; Antonova, E. S.; Zhuravlev, A. A. Study of Reaction-Bonded Boron Carbide Properties. Refract. Ind. Ceram. 2015, 55, 414418.

(31) Mukhanov, V. A.; Kurakevych, O. O.; Solozhenko, V. L. The Interrelation between Hardness and Compressibility of Substances and Their Structure and Thermodynamic Properties. J. Superhard Mater. 2009, 30, 368-378. 
(32) Lazzari, R.; Vast, N.; Besson, J. M.; Baroni, S.; Dal Corso, A. Atomic Structure and Vibrational Properties of Icosahedral B4C Boron Carbide. Phys. Rev. Lett. 1999, 83, $3230-3233$.

(33) Niihara, K.; Nakahira, A.; Hirai, T. The Effect of Stoichiometry on Mechanical Properties of Boron Carbide. J. Am. Ceram. Soc. 1984, 67, C13-C14.

(34) Mingos, D. M. P. A General Theory for Cluster and Ring Compounds of the Main Group and Transition Elements. Nat. Phys. Sci. 1972, 236, 99-102.

(35) Wade, K. The Structural Significance of the Number of Skeletal Bonding Electron-Pairs in Carboranes, the Higher Boranes and Borane Anions, and Various Transition-Metal Carbonyl Cluster Compounds. J. Chem. Soc. D Chem. Commun. 1971, 15, 792-793.

(36) An, Q.; Goddard III, W. A.; Cheng, T. Atomistic Explanation of Shear-Induced Amorphous Band Formation in Boron Carbide. Phys. Rev. Lett. 2014, 113, 95501.

(37) Mondal, S. Charge Transfer and Fractional Bonds in Stoichiometric Boron Carbide. Chem. Mater. 2017, 29, 6191-6194.

(38) Aselage, T. L.; Tissot, R. G. Lattice Constants of Boron Carbides. J. Am. Ceram. Soc. 1992, 75, 2207-2212.

(39) Kresse, G.; Hafner, J. Ab Initio Molecular Dynamics for Liquid Metals. Phys. Rev. B 1993, $47,558-561$.

(40) Kresse, G.; Furthmüller, J. Efficient Iterative Schemes for Ab Initio Total-Energy Calculations Using a Plane-Wave Basis Set. Phys. Rev. B 1996, 54, 11169-11186.

(41) Kresse, G.; Furthmüller, J. Efficiency of Ab-Initio Total Energy Calculations for Metals 
and Semiconductors Using a Plane-Wave Basis Set. Comput. Mater. Sci. 1996, 6, 15-50.

(42) Kresse, G. From Ultrasoft Pseudopotentials to the Projector Augmented-Wave Method. Phys. Rev. B 1999, 59, 1758-1775.

(43) Silvi, B.; Savin, A. Classification of Chemical-Bonds Based on Topological Analysis of Electron Localization Functions. Nature 1994, 371, 683-686.

(44) Becke, A. D.; Edgecombe, K. E. A Simple Measure of Electron Localization in Atomic and Molecular Systems. J. Chem. Phys. 1990, 92, 5397-5403.

(45) Page, Y. Le; Saxe, P. Symmetry-General Least-Squares Extraction of Elastic Data for Strained Materials from Ab Initio Calculations of Stress. Phys. Rev. B 2002, 65, 104104.

(46) Hill, R. The Elastic Behaviour of a Crystalline Aggregate. Proc. Phys. Soc. Sect. A 1952, $65,349-354$.

(47) Roundy, D.; Krenn, C.; Cohen, M.; Morris, J. Ideal Shear Strengths of Fcc Aluminum and Copper. Phys. Rev. Lett. 1999, 82, 2713-2716.

(48) Li, B.; Sun, H.; Chen, C. Large Indentation Strain-Stiffening in Nanotwinned Cubic Boron Nitride. Nat. Commun. 2014, 5, 4965.

(49) An, Q.; Goddard III, W. A. Nanotwins Soften Boron-Rich Boron Carbide (B13C2). Appl. Phys. Lett. 2017, 110, 111902. 


\section{TOC Graphic}

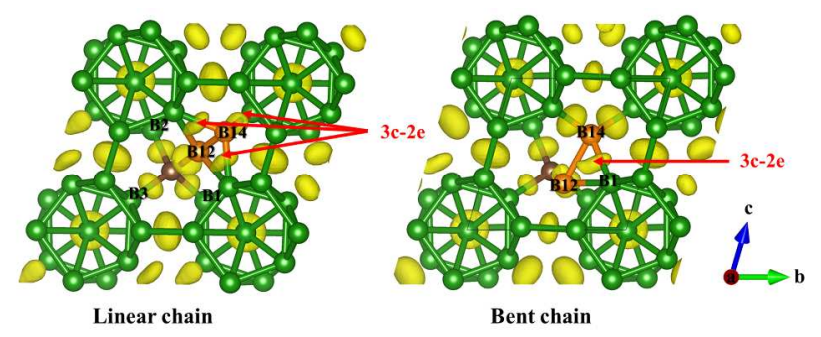

